\section{GOBIERNO CORPORATIVO EN PYMES DEL MERCADO \\ DE CAPITALES ARGENTINO: ANÁLISIS DE LOS PANELES DE COTIZACIÓN*}

\author{
CORPORATE GOVERNANCE IN ARGENTINEAN SMES: \\ ANALYSIS OF THE QUOTATION PANELS
}

\section{Anahí Briozzo | Diana Albanese | Matías Villegas}

\section{RESUMEN}

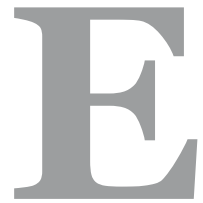

l objetivo de este trabajo es estudiar las características de gobierno corporativo en las pymes emisoras de títulos en el mercado de capitales de la Argentina, en dos sectores de negociación: régimen general y régimen pyme. La existencia de diferencias significativas entre ambos grupos de interés se estudia en forma bivariada mediante el test de Pearson Exact para variables binarias y test de Kruskal Wallis para las variables continuas. Posteriormente se analiza la robustez de las variables significativas empleando una regresión de mínimos cuadrados ordinarios con variables control. Las empresas bajo ambos regímenes no presentan diferencias en cuando a la conformación accionaria, dualidad del CEO, características del auditor externo, y participación de mujeres en la propiedad, directorio y alta gerencia. La dispersión accionaria es baja y existe una elevada concentración de la propiedad en el principal accionista directo. La participación de mujeres es baja en la propiedad, el directorio y la alta gerencia. Como diferencia significativa se observa que en el régimen general las empresas presentan directorios y cuadros gerenciales con un promedio de un integrante más.

Palabras clave: gobierno corporativo, pymes, mercado de capitales.

\section{ABSTRACT}

The aim of this paper is to study the characteristics of corporate governance in small and medium enterprises (SMEs) issuing securities in the capital markets of Argentina, in two sectors of negotiation: general regime and SME regime. The existence of significant differences between

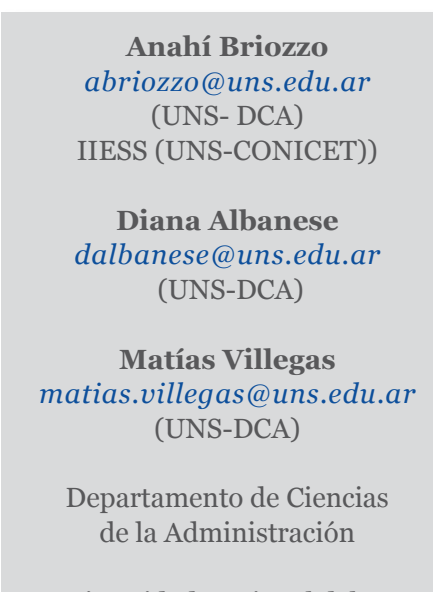

Universidad Nacional del Sur ARGENTINA

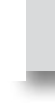

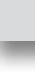


both interest groups is studied in a bivariate way using the Pearson Exact test for binary variables and the Kruskal Wallis test for continuous variables. Subsequently, the robustness of the significant variables is analyzed using a regression of ordinary least squares with control variables. The companies under both regimes do not present differences in terms of shareholder structure, CEO duality, characteristics of the external auditor, and participation of women in the property, board of directors and senior management. The share dispersion is low and there is a high concentration of ownership in the main direct shareholder. The participation of women is low in the property, the board of directors and senior management. As a significant difference, it can be observed that in the general regime, companies present boards and management teams with an average of one more member.

Keywords: corporate governance, SMEs, capital markets.

\section{INTRODUCCIÓN}

El gobierno corporativo se ha convertido en un tema de interés dentro de las organizaciones, en los mercados financieros, y se constituye un tema de estudio de relevancia. Diversos estudios (Campos, 2002; Bauer,2002; Foerster \& Huen, 2004; CEF, 2005; Bebczuk, 2005) muestran consenso en cuanto a que el buen GC tiene relación con el valor de mercado de la empresa y alienta la estabilidad y desarrollo del sistema financiero, lo que lleva a las empresas a obtener crecimiento económico, acceso a financiamiento y mejores rendimientos.

La temática de GC ha sido ampliamente estudiada en países desarrollados y en grandes empresas, pero existen pocos antecedentes en países emergentes en general, y en particular en firmas de pequeño tamaño, a pesar de la relevancia de las mismas para el desarrollo económico.

El objetivo de este trabajo es estudiar las características de gobierno corporativo (GC) en las pymes emisoras de títulos (acciones y/o obligaciones negociables) en el mercado de capitales de la Argentina. El mercado de capitales argentino ofrece dos sectores donde las pymes pueden ofrecer sus títulos: el régimen general, y el panel pyme, con menores requisitos de registración e información, y participación limitada a inversores calificados. Si los diferentes requerimientos de ambos paneles tienen efecto en el desarrollo de GC de las pymes es un tema que aún no ha sido abordado.

Según informa la CNV (2018), a marzo de 2018, el 31\% de las empresas bajo régimen de oferta pública son pymes. Asimismo, el financiamiento bancario recibido por las pymes durante el primer trimestre de 2018 se incrementó 23\% respecto del mismo período el año previo, mientras que el financiamiento en el mercado de capitales lo hizo un 57\%, llevando su participación en el total del 5\% al 6\% (CNV 2018).

*Este trabajo ha sido desarrollado en el marco del proyecto (PGI-UNS) "Desafíos del gobierno corporativo en empresas argentinas: su relación con la rentabilidad, financiamiento y riesgo" (24/C049). 
En función de lo expuesto anteriormente, la pregunta de investigación que motiva este trabajo es: ¿Difieren las características de GC difieren entre empresas emisoras bajo el régimen pyme, y pymes del panel general?

\section{MARCO TEÓRICO DE REFERENCIA}

Existen múltiples definiciones del término GC. La definición tradicional es la que relaciona al GC con la protección de los intereses de los accionistas (Tirole, 2001) y tiene su origen en los problemas surgidos como consecuencia de la separación entre la propiedad y el control (Berle \& Means, 2017). Dichos problemas se enmarcan dentro la Teoría de la Agencia y encuentran al GC como un intento de disminuir las consecuencias adversas de las acciones y decisiones de los administradores, evitar el riesgo moral y los beneficios privados del control. Sin embargo, hoy es ampliamente aceptada la extensión del concepto hacia la protección de los intereses de otras partes involucradas en la firma (stakeholders: acreedores, clientes, proveedores, empleados, comunidad, etc.), tal como queda expresado en la definición propuesta por la Organización para la Cooperación y Desarrollo Económico:

"El GC es el sistema por el que las corporaciones de negocios son dirigidas y controladas. La estructura de GC especifica la distribución de derechos y responsabilidades entre los diferentes participantes en la corporación, como el directorio, la gerencia, los accionistas y otros incumbentes (stakeholders) y detalla las reglas y procedimientos para la toma de decisiones sobre los temas corporativos. Para hacer esto, también provee la estructura a través de la cual se determinan los objetivos de la compañía, se asignan los medios para alcanzar dichos objetivos y se determina cómo se monitorea el desempeño en relación a los objetivos perseguidos".

Casal (2010) define el concepto de GC como un conjunto de principios, políticas, procedimientos, estándares y normas que se utilizan para dirigir y controlar una entidad de cualquier naturaleza, tamaño o condición, de una forma ética equitativa y responsable.

La importancia de un adecuado GC radica en ofrecer un conjunto de condiciones que garanticen a los diferentes stakeholders que podrán recuperar su inversión, más alguna remuneración por ella.

Según señalan Chisari \& Ferro (2012) los Principios de la OCDE sobre el buen GC han ejercido importante influencia intelectual y de diseño de políticas. Su primera versión fue aprobada en 1999, con una reforma en el 2004 y 2015, y abarca los siguientes puntos: 1) Asegurar las bases de un marco efectivo de gobierno corporativo, 2) Derechos de los accionistas y funciones claves de la propiedad, 3) Trato equitativo a los accionistas, 4) Rol de los stakeholders en el gobierno corporativo, 5) Diseminación de información y transparencia, y 6) Responsabilidades del directorio. 
Las mejores prácticas de GC incluyen un componente institucional y otro contractual. El marco legal y normativo es conocido como GC institucional. Por su parte, las iniciativas y los compromisos que voluntariamente decidan adoptar las empresas por encima de los requisitos legales se conocen como GC contractual. De acuerdo con lo expresado por el Centro para la Estabilidad Financiera (CEF, 2005) la correlación positiva entre el GC contractual y el valor de la empresa es más fuerte en los mercados emergentes que en los mercados desarrollados, a pesar de que estos últimos cuentan con un mejor marco institucional. Es justamente en contextos institucionales relativamente más débiles donde los compromisos contractuales asumidos por las propias empresas generan un mayor beneficio para las mismas.

No existe un modelo único de GC. La estructura a adoptar depende del modelo de gestión empresarial que adopte la empresa. Existe una clasificación preliminar de dos modelos extremos. Uno se relaciona con el que se adopta en países donde el sistema legal se enmarca dentro del common law, denominado angloamericano y está orientado hacia los accionistas. El otro, se refiere al modelo adoptado por países con un sistema legal dentro del civil law y se denomina euroasiático. El primero, denominado también "Modelo Basado en el Mercado", se caracteriza por una alta atomización de la propiedad, un mercado de capitales eficiente y desarrollado, y por un "mercado de control" muy activo donde las compras hostiles (takeovers), las reestructuraciones y fusiones forman parte del escenario corporativo. Por su parte, el modelo euroasiático, también conocido como “Modelo Basado en Relaciones", es el modelo corporativo prevaleciente en Europa, Japón, América Latina y, en general, en el resto de los países fuera del mundo anglosajón. Está basado en sólidas relaciones de la firma con ciertos accionistas controlantes, en general, bancos o conglomerados (holdings empresarios), y con financiamiento mucho menos dependiente del mercado de capitales que de los bancos, y un mercado de control casi inexistente.

Por otra parte, Gillian (2006) y Brown, Beekes \& Verhoeven (2011) distinguen entre características internas y externas del GC. Las características internas son las estructuras y procesos del GC que están bajo el control de los accionistas y del Directorio. En cambio, los factores exógenos tienen relación con los conflictos de agencia y sus costos, e incluyen el sector al cual pertenece la empresa, sus oportunidades futuras de inversión, sus recursos y tecnología, el sistema legal, los estándares contables, los mercados de capitales y sus reglas de operación, entre otros.

\subsection{Antecedentes empíricos en pymes}

La literatura empírica sobre GC en pymes se ha desarrollado mayoritariamente en países desarrollados. Abor \& Adjasi (2007) indican que los mecanismos de GC en pymes a los que se les presta más atención son: separación de alta gerencia y directorio, para asegurar el rol de control de éste último, combinación adecuada de directores independientes e internos e independencia del auditor externo para asegurar la integridad de los reportes financieros. Resulta interesante 
mencionar que, si bien los mecanismos de GC podrían ser en principio similares a los de las grandes empresas, la evidencia empírica en pymes no es homogénea.

De acuerdo a Aguirre \& de las Carreras (2011) ${ }^{1}$, las prácticas relativas a Gobierno Corporativo en la Argentina, deberían orientarse a dos temas principales, (1) Principio de voluntariedad: la adopción debería ser plenamente voluntaria para las Emisoras, si ellas apreciaran un efecto positivo y (2) Creación de los incentivos adecuados para la adopción voluntaria de los principios de buen gobierno societario en el mercado de capitales local. Plantean que la experiencia del Nuevo Mercado de Brasil puede ser positiva en este sentido.

Brunninge, Nordqvist \& Wiklund (2007) encuentran que en las pymes suecas con mayor concentración de la propiedad la tendencia al cambio estratégico es menor, lo cual estaría asociado con la aversión al riesgo y la resistencia al cambio. La presencia de directores externos y el número de miembros de la alta gerencia tienen un efecto positivo sobre los cambios estratégicos, y estos dos mecanismos actúan en cierto grado como sustitutos entre sí.

Tauringana (2015) estudia las pymes listadas en el UK Alternative Investment Market por un periodo de 10 años, encontrando que el tamaño del directorio tiene un efecto negativo en la $Q$ de Tobin, mientras que la participación de directores no ejecutivos no tiene efecto significativo. Al-Najjar \& Al-Najjar (2017) estudian pymes de capital abierto de Reino Unido y construyen un índice de GC basado en diez variables, que comprenden características y funcionamiento del directorio. Encuentran que mayores niveles de GC se encuentran relacionados positivamente con el tamaño, la Q de Tobin, y en forma inversa con el endeudamiento de la empresa.

Arosa, Iturralde y Maceda (2013) reportan que el tamaño del directorio y el porcentaje de directores externos inciden negativamente en el rendimiento de las pymes españolas. Estos resultados indicarían los efectos de la falta de coordinación, flexibilidad y comunicación en un directorio de gran tamaño. Para empresas no listadas, Hernández-Cánovas, Mínguez-Vera y Sánchez-Vidal (2016) analizan la relación entre la estructura de propiedad y el endeudamiento de pymes españolas. Encuentran que la presencia de un individuo como accionista principal tiene un efecto positivo en la deuda, mientras que un accionista principal que sea una sociedad tiene un efecto negativo. La concentración de propiedad en manos del principal accionista tiene un efecto negativo en el apalancamiento.

Por otro lado, Dasilas y Papasyriopoulos (2015) comparan GC en pymes y grandes empresas griegas listadas encuentran que el tamaño del directorio se relaciona positivamente con el nivel y plazo del endeudamiento. Un auditor Big 4 también tiene un efecto positivo en el endeudamiento. Durante el periodo de crisis en Grecia la independencia del directorio es la variable más relevante que explica el endeudamiento.

En países emergentes, Abor (2007) encuentra para una muestra de pymes no listadas de Ghana que el tamaño y composición del directorio, la profesionalización de la gerencia, la duali- 
dad del CEO y la estructura de propiedad (propietarios-gerentes, empresa familiar y accionistas extranjeros), afectan positivamente a la rentabilidad de la empresa.

\subsection{Antecedentes empíricos en la Argentina}

En la Argentina, el CEF (2005) presenta un estudio que abarca un período de observación entre 2000-2003, relacionando medidas contables (rendimiento operativo y patrimonial) y medidas de mercado (Q de Tobin, Precio/Valor libros) con el Índice de Transparencia y Difusión (ITD), arrojando como resultado un efecto cuantitativo muy apreciable: un incremento de diez puntos porcentuales a partir del ITD promedio para la muestra se traduciría en un aumento del rendimiento operativo de 2,6 puntos porcentuales, además se encuentra una relación positiva y robusta entre el nivel de GC (medido a través del ITD) y el valor de la Q de Tobin.

Bebczuck (2005) sobre una muestra de 65 empresas no financieras listadas en el Mercado de Valores de Buenos Aires S.A. encuentra una relación positiva y robusta entre el ITD y el rendimiento operativo y la Q de Tobin, durante el período 2000-2003. Sin embargo, dado que ITD solo mide aquello que las empresas voluntariamente deciden difundir, el autor propone un índice que combina tres variables binarias: (1) participación de la acción en carteras de fondos de pensión; (2) aceptación de la empresa a contestar la encuesta sobre GC elaborada por el propio autor; y (3) porcentaje de directores independientes por encima de la media de la muestra. Dicho índice exhibió una correlación de 0,4 con el ITD. La regresión del nuevo índice contra ROA y Q fue significativa y positiva para el período bajo análisis. Briozzo et al. (2008) describen la situación del GC en la Argentina, destacando no solo las falencias en cuanto al GC institucional, sino también su escaso cumplimiento (enforcement).

Chisari y Ferro (2012) desarrollan una aplicación empírica de mejores prácticas de gobierno corporativo en la Argentina usando un modelo de equilibrio general computable. Encuentran que la implementación de la reforma tiene un costo para la economía de una reducción del crecimiento del PBI, y que si se considera una reducción del costo de capital de las empresas como consecuencia del buen GC, primero, aumenta el valor de las empresas, y segundo, si es anticipada, favorece a la acumulación de capital y mejora la asignación de recursos.

Para pymes listados en la Argentina, Briozzo, Albanese y Santoliquido (2017) analizan el panel pyme del Mercado de Valores de Buenos Aires y encuentran que existen relaciones significativas entre la participación de mujeres en la propiedad y la auditoría externa, y las decisiones de financiamiento.

En un estudio comparativo entre pymes emisoras de la Argentina y España, se observa que la participación de las corporaciones en la propiedad (indicador de grupo económico) y un auditor Big4 tienen una relación negativa con el ratio de pasivos a corto plazo para ambos países (Briozzo, Cardone-Riportella y García-Olalla, 2019). 


\section{METODOLOGÍA}

La población bajo estudio consiste en las pymes emisoras de títulos en el mercado de capitales argentino. De un total de 88 empresas a abril 2018, 40 pertenecen al régimen general y 48 al régimen pyme (CNV 2017). Cabe destacar que las pymes están exceptuadas de la presentación del código de gobierno societario ${ }^{2}$, por lo cual la información de interés no se encuentra reunida en un único documento.

Por lo tanto, a partir de la información disponible en forma pública en el sitio web de la CNV (estados financieros, prospectos de emisión, hechos relevantes, entre otros) se desarrolló una base ad hoc de datos de pymes emisoras de títulos negociables con la última información disponible a abril 2018. La información recabada incluye composición y funcionamiento del directorio, estructura de propiedad y administración, características de la empresa auditora. Dado que una de las fuentes más completas de información de GC son los prospectos de emisión de títulos, se encontró que para las pymes bajo régimen general existen pocos datos disponibles, reduciéndose la muestra de estas empresas a 12 casos para este conjunto de variables. En las empresas estudiadas encontramos solo cuatro emisoras de acciones, siendo el resto emisoras sólo de obligaciones negociables.

La existencia de diferencias significativas entre ambos grupos de interés se estudia en forma bivariada mediante el test de Pearson Exact para variables binarias y test de Kruskal Wallis para las variables continuas. Posteriormente se estudia la robustez de las variables significativas empleando una regresión de mínimos cuadrados ordinarios (MCO) con variables control. Las variables estudiadas y su definición operativa se presentan en la tabla 1.

Tabla 1: Definiciones operativas de las variables

\begin{tabular}{|l|l|}
\hline Variables GC & Definición \\
\hline Nro. Accionistas & Número de accionistas con participación mayor al 5\% \\
\hline \% Personas Jurídicas Accionistas & Accionistas sociedades/Número accionistas \\
\hline Participación del ppal. accionista & Tenencia accionaria del principal accionista directo \\
\hline Ppal. Accionista mujer & Variable binaria, toma valor 1 cuando el principal accionista es mujer. \\
\hline$\%$ mujeres accionistas & Mujeres accionistas/ Accionistas personas físicas \\
\hline Tenencia accionaria mujeres & Participación accionaria de todas las accionistas mujeres \\
\hline Nro Miembros tit. Directorio & $\begin{array}{l}\text { Número de integrantes titulares del directorio. Mínimo } 3 \text { integrantes } \\
\text { (Art. 255 Ley General de Sociedades) }\end{array}$ \\
\hline \% de directores externos & $\begin{array}{l}\text { Directores que no son accionistas ni parte del cuadro gerencial/ número } \\
\text { total de directores (titulares y suplentes) }\end{array}$ \\
\hline$\%$ directoras mujeres & Directoras mujeres/ Número total de directores \\
\hline Dualidad CEO & $\begin{array}{l}\text { Variable binaria, toma valor } 1 \text { cuando el CEO y el presidente del directorio } \\
\text { son la misma persona }\end{array}$ \\
\hline Nro. Personas alta gerencia & Número de personas informadas en el cuadro gerencial \\
\hline$\%$ mujeres alta gerencia & Mujeres en cuadro gerencial/Número de personas alta gerencia \\
\hline
\end{tabular}

\footnotetext{
${ }^{2}$ La Res. CNV 606/12 establecía los requerimientos para la presentación del código de gobierno societario vigentes al momento del estudio, y ha sido recientemente reemplazada por la Res. 797/19. Esta norma es de aplicación obligatoria para los estados financieros cerrados a partir del 31 de diciembre de 2019. La nueva resolución mantiene la excepción para las pymes.
} 


\begin{tabular}{|l|l|}
\hline Variables GC & Definición \\
\hline Auditor Big4 & $\begin{array}{l}\text { Variable binaria, tomar valor } 1 \text { cuando la empresa auditora del último } \\
\text { EEFF es PWC, KPMG, Deloitte o Ernst \& Young }\end{array}$ \\
\hline Rotación auditor & $\begin{array}{l}\text { Variable binaria, tomar valor } 1 \text { cuando el auditor externo ha cambiado en } \\
\text { los últimos tres años }\end{array}$ \\
\hline Variables control & Antigüedad a abril 2018 \\
\hline Antigüedad & Número de empleados año 2017 \\
\hline Nro de empleados & Ventas último EEFF en millones de \$ \\
\hline Ventas & $\begin{array}{l}\text { Variable binaria, toma valor 1 cuando la empresa cotiza bajo el régimen } \\
\text { general }\end{array}$ \\
\hline Variable régimen de cotización &
\end{tabular}

Fuente: Elaboración propia.

\section{RESULTADOS}

En la Tabla 2 se presenta la estadística descriptiva de la muestra, donde se observa que el la dispersión accionaria es baja, que existe una elevada concentración de la propiedad en el principal accionista directo, quien en muy pocas ocasiones es mujer. La participación de mujeres también es baja en la propiedad, el directorio y la alta gerencia. El tamaño promedio del directorio supera levemente el mínimo normativo de tres integrantes, mientras que la dualidad del CEO está presente en la mitad de las empresas. Con respecto al auditor externo es escasa la contratación de los Big 4, y también es baja la rotación del mismo.

Tabla 2: Estadística descriptiva

\begin{tabular}{|l|c|c|c|c|}
\hline & Media & Desvío & Min & Max \\
\hline Nro. Accionistas & 3,56 & 2,09 & 2 & 13 \\
\hline$\%$ Personas Jurídicas Accionistas & $27 \%$ & $45 \%$ & 0 & $100 \%$ \\
\hline Participación del ppal. accionista & $57 \%$ & $23 \%$ & $23 \%$ & $100 \%$ \\
\hline Ppal. Accionista mujer & $4 \%$ & $19 \%$ & 0 & $100 \%$ \\
\hline$\%$ mujeres accionistas & $24 \%$ & $28 \%$ & o & $100 \%$ \\
\hline Tenencia accionaria mujeres & $9 \%$ & $15 \%$ & 0 & $75 \%$ \\
\hline Nro Miembros tit. Directorio & 3,84 & 1,02 & 3 & 8 \\
\hline$\%$ de directores externos & $45 \%$ & $37 \%$ & 0 & $100 \%$ \\
\hline$\%$ directoras mujeres & $16 \%$ & $20 \%$ & 0 & $67 \%$ \\
\hline Dualidad CEO & $48 \%$ & $50 \%$ & 0 & $100 \%$ \\
\hline Nro. Personas alta gerencia & 4,19 & 2,54 & 0 & 13 \\
\hline$\%$ mujeres alta gerencia & $19 \%$ & $24 \%$ & 0 & $100 \%$ \\
\hline Auditor Big4 & $6 \%$ & $23 \%$ & 0 & $100 \%$ \\
\hline Rotación auditor & $18 \%$ & $39 \%$ & 0 & $100 \%$ \\
\hline
\end{tabular}




\begin{tabular}{|l|c|c|c|c|}
\hline Variables control \\
\hline Antigüedad (años) & 26,66 & 18,13 & 5 & 95 \\
\hline Nro de empleados & 127,56 & 209,70 & 2 & 1.337 \\
\hline Ventas ( millones de \$) & 284,00 & 327,00 & 0,43 & 1.830 \\
\hline
\end{tabular}

Fuente: Elaboración propia.

La Tabla 3 muestra los resultados del análisis bivariado para las variables de interés. Las pymes del régimen general son más antiguas, y poseen directorios y cuadros gerenciales de mayor tamaño. Las pymes de ambos paneles poseen características similares en cuanto a nivel de ventas, estructura de propiedad (considerando accionistas con más de $5 \%$ de tenencia), características del auditor externo y participación de mujeres en la propiedad, dirección y administración.

Tabla 3: Caracterización de empresas según panel de cotización

\begin{tabular}{|l|c|c|c|c|}
\hline Variables GC & Reg. general & Reg. pymes & p-value & Test \\
\hline Nro. Accionistas & 4,3 & 3,3 & 0,14 & KW \\
\hline \% Personas Jurídicas Accionistas & $25 \%$ & $28 \%$ & 0,83 & KW \\
\hline Participación del ppal. accionista & $58 \%$ & $56 \%$ & 0,44 & KW \\
\hline Ppal. Accionista mujer & $0 \%$ & $4,90 \%$ & 0,43 & FE \\
\hline \% Mujeres accionistas & $25 \%$ & $24 \%$ & 0,54 & KW \\
\hline Tenencia accionaria mujeres & $8,30 \%$ & $9,80 \%$ & 0,35 & KW \\
\hline Nro. Miembros Tít. Directorio & 4,67 & 3,61 & 0,004 & KW \\
\hline \% De directores externos & $47 \%$ & $44 \%$ & 0,78 & KW \\
\hline$\%$ Directoras mujeres & $19 \%$ & $15 \%$ & 0,14 & KW \\
\hline Dualidad CEO & $44 \%$ & $49 \%$ & 0,56 & PE \\
\hline Nro. Personas alta gerencia & 5,7 & 3,8 & 0,009 & KW \\
\hline \% Mujeres alta gerencia & $23 \%$ & $17 \%$ & 0,42 & KW \\
\hline Auditor Big4 & $10 \%$ & $2 \%$ & 0,134 & PE \\
\hline Rotación auditor & $9 \%$ & $20 \%$ & 0,35 & PE \\
\hline Variables control & \multicolumn{5}{|l|}{} \\
\hline Antigüedad & 34,3 & 23,7 & 0,09 & KW \\
\hline Nro. de empleados & 137,7 & 124,1 & 0,39 & KW \\
\hline Ventas & 292 & 278 millones de $\$$ & 0,23 & KW \\
\hline
\end{tabular}

Nota: Test de Kruskal-Wallis (KW) para variables continuas y Pearson Exact (PE) para variables binarias. Fuente: Elaboración propia.

A fines de evaluar la robustez de las diferencias observadas en el directorio y la alta gerencia, se presentan en la Tabla 4 las regresiones múltiples de estas variables empleando variables control. Se puede observar que aun controlando por tamaño bajo diferentes definiciones, las pymes que cotizan bajo el régimen general tienen un directorio en promedio con un integrante titular más (M1 a M3), y un cuadro de alta gerencia con 1,5-1,8 miembros más (M5 y M6). 
Tabla 4: Regresiones MCO para variables dependientes directores titulares y alta gerencia

\begin{tabular}{|c|c|c|c|c|c|c|}
\hline \multirow{2}{*}{ Variable } & \multicolumn{3}{|c|}{$\mathrm{Y}=$ Nro. Directores titulares } & \multicolumn{3}{|c|}{$\mathrm{Y}=$ Nros. personas alta gerencia } \\
\hline & M1 & M2 & M3 & M4 & M5 & M6 \\
\hline Régimen Gral. & $\begin{array}{l}1,007^{* * *} \\
(0,003)\end{array}$ & $\begin{array}{l}1,104^{* * * *} \\
(0,002)\end{array}$ & $\begin{array}{l}1,093^{* * *} \\
(0,000)\end{array}$ & $\begin{array}{l}1,436 \\
(0,134)\end{array}$ & $\begin{array}{l}1,815^{* *} \\
(0,027)\end{array}$ & $\begin{array}{c}1,490^{*} \\
(0,087)\end{array}$ \\
\hline Antigüedad & $\begin{array}{c}0,003 \\
(0,679)\end{array}$ & & & $\begin{array}{l}0,025 \\
(0,214)\end{array}$ & & \\
\hline Empleados & & $\begin{array}{c}-0,0003 \\
(0,705) \\
\end{array}$ & & & $\begin{array}{c}0,001 \\
(0,473) \\
\end{array}$ & \\
\hline Log(Ventas) & & & $\begin{array}{c}0,192^{* * *} \\
(0,033)\end{array}$ & & & $\begin{array}{l}0,663^{* * *} \\
(0,029)\end{array}$ \\
\hline Constante & $\begin{array}{l}3,540 \\
(0,000)\end{array}$ & $\begin{array}{c}3,753 \\
(0,000)\end{array}$ & $\begin{array}{l}-0,041 \\
(0,981)\end{array}$ & $\begin{array}{l}3,171 \\
(0,000)\end{array}$ & $\begin{array}{c}3,554 \\
(0,000)\end{array}$ & $\begin{array}{l}-8,813 \\
(0,123)\end{array}$ \\
\hline Prob. $>$ F & 0,005 & 0,001 & 0,001 & 0,048 & 0,058 & 0,009 \\
\hline R2 ajustado & 0,160 & 0,170 & 0,150 & 0,087 & 0,101 & 0,150 \\
\hline
\end{tabular}

Nota: P-values entre paréntesis. Se denota con ${ }^{* * *}$ significatividad al 1\%, ${ }^{* *}$ al $5 \%$, * al $10 \%$. Fuente: Elaboración propia.

\section{CONCLUSIONES}

Los resultados de esta investigación señalan las diferencias existentes en el desarrollo del GC en pymes sujetas a diferentes regímenes de cotización en la Argentina. Sólo cuatro empresas han emitido acciones a abril de 2018, mientras que el resto cotiza solo obligaciones negociables. Los resultados muestran que en el régimen general las empresas presentan directorios y cuadros gerenciales con un integrante más. Es interesante mencionar que esta diferencia se mantiene aun controlando por tamaño y antigüedad.

Se destaca la heterogeneidad en la disponibilidad de información sobre GC en las pymes de ambos paneles, dado que al estar exceptuadas de presentar el Código de Gobierno Societario (Res CNV 606/12), la información se encuentra disponible en distintos documentos, y muchas veces acotada a los prospectos de emisión más recientes. A fines de lograr mayor transparencia y uniformar el acceso a la información sobre GC sería deseable la presentación de información periódica simplificada para las pymes independientemente del sector de cotización.

Las limitaciones de este trabajo radican en las restricciones de acceso a la información sobre GC de las pymes, en especial para el panel general. Se presenta como futura línea de investigación la actualización continua de la base de datos a fines de realizar estudios de evolución temporal. 


\section{REFERENCIAS BIBLIOGRÁFICAS}

Abor, J., \& Adjasi, C. K. (2007). Corporate governance and the small and medium enterprises sector: theory and implications. Corporate Governance: The international journal of business in society, 7(2), 111-122.

Al-Najjar, B. \& Al-Najjar , D. (2017). The impact of external financing on firm value and a corporate governance index: SME evidence. Journal of Small Business and Enterprise Development, 24 (2), 411-423.

Arosa, B., Iturralde, T. \& Maseda, A. (2013). The board structure and firm performance in SMEs: Evidence from Spain. Investigaciones Europeas de Dirección y Economía de la Empresa, 19, 127-135.

Bauer, R., Günster, N. \& Otten, R. (2003). Empirical Evidence on Corporate Governance in Europe. Journal on Asset Management, 5, 91-104.

Bebczuck, R. (2005). Corporate Governance and Owenship: Measurant and Impact on Corporate Performance in Argentina. En Chong, A., \& Lopez de Silanes, F. (Eds.). (2007). Investor protection and corporate governance: Firmlevel evidence across Latin America. The World Bank. Standorf University Press.

Berle, A. A., \& Means, G. C. (2017). Modern corporation and private property. Segunda edición. Routledge, Nueva York.

Briozzo, A, Albanese, D. \& Santoliquido, D., (2017). Corporate governance, financing and gender: A study of SMEs from Argentinean Securities Markets. Contaduría y Administración, 62(2), 358-376.

Briozzo, A., Cardone-Riportella, C., \& García-Olalla, M. (2019). Corporate governance attributes and listed SMES'debt maturity. Corporate Governance: The International Journal of Business in Society. https://doi.org/10.1108/CG-11-2017-0272

Brown, P., Beekes, W. \& Verhoeven, P. (2011). Corporate governance, accounting and finance: A review. Accounting and Finance, 51, 96-172. 
Brunninge, O., Nordqvist, M., \& Wiklund, J. (2007). Corporate Governance and Strategic Change in SMEs: The Effects of Ownership, Board Composition and Top Management Teams. Small Business Economics, 29, 295-308.

Campos, C., Newell, R., \& Wilson, G. (2002). Corporate Governance Develops in Emerging Markets: Shareholders in Emerging Markets Show They're Willing to Pay a Premium for Good Governance Standards. McKinsey on Finance, 3, 15-18.

Casal A.M. (2010). Gobierno Corporativo. Dirección, Administración y Control de Organizaciones en Forma Ética. Ed. Errepar Buenos Aires.

Centro para la Estabilidad Financiera (CEF) (2005): Nota de Política No 5 "El Gobierno Corporativo en Argentina”. Julio 2005.

Chisari, O. \& Ferro, G. (2012). "Una contribución a la evaluación de las reformas al Gobierno Corporativo de las empresas: aplicación a la Argentina”. HAL Id: hal-o0694556 https://hal.archives-ouvertes.fr/hal-00694556

Comisión Nacional de Valores. (2012). Resolución General 606/2012. Modificación Normas CNV (N.T. 2001) - Código de Gobierno Societario. Disponible en: http://www.cnv.gob.ar/LeyesyReg/CNV/esp/RGCRGN6o6-12.htm

Comisión Nacional de Valores (2017). Informe Trimestral de Financiamiento a pymes. Cuatro trimestre 2017. Disponible en: http://www.cnv.gov.ar/sitioWeb/Informes

Comisión Nacional de Valores (2018). Informe Trimestral de Financiamiento a pymes. Primer trimestre 2018. Disponible en: http://www.cnv.gov.ar/sitioWeb/Informes

Comisión Nacional de Valores (2019). Resolución General 797/19. Reforma del Código de Gobierno Societario. Disponible en:

https://www.boletinoficial.gob.ar/detalleAviso/primera/209844/20190619

Dasilas, A., \& Papasyriopoulos, N. (2015). Corporate governance, credit ratings and the capital structure of Greek SME and large listed firms. Small Business Economics, 45(1), 215-244.

Foerster, S. \& Huen, B. (2004). Does Corporate Governance Matter to Canadian Investors?. Canadian Investors Review, 17, 19-25. 
Gillian, S. (2006). Recent Developments in Corporate Governance: An Overview. Journal of Corporate Finance, 12, 381-402.

Hernández-Cánovas, G., Mínguez-Vera, A. \& Sánchez-Vidal (2016). Ownership structure and debt as corporate governance mechanisms: an empirical analysis for Spanish smes, Journal of Business Economics and Management, 17(6), 960-976.

OCDE (2015): G20/OECD Principles of Corporate Governance. Disponible en:

http://www.oecd-ilibrary.org/governance/g20-oecd-principles-of-corporate-governance2015_9789264236882-en

Tauringana, G. (2015). Corporate governance and performance of UK listed small and medium enterprises. Corporate Governance, 15 (5), 719 - 733.

Tirole, J. (2001): “Corporate Governance”. Econometrica, 69, 1-35.

\section{COMO CITAR ESTE ARTÍCULO}

BRIOZZO, Anahí, ALBANESE, Diana y VILLEGAS, Matías. Gobierno corporativo en pymes del mercado de capitales argentino: Análisis de los paneles de cotización. Revista de la Facultad de Ciencias Económicas - UNNE, Argentina. Volumen 22, Núm. 1, enero-junio 2019, ISSN: 1668 - 6365. Págs. 93 - 106. DOI: http://dx.doi.org/10.30972/rfce.2213950

\section{CURRICULUM VITAE}

\section{Anahí Briozzo}

Licenciada en Administración y Doctora en Economía (UNS). Profesora titular del Departamento de Ciencias de la Administración de la Universidad Nacional del Sur (DCA-UNS) e Investigadora Adjunta del IIESS (UNS-CONICET), Bahía Blanca, Argentina. Docente investigador categoría 2. abriozzo@uns.edu.ar 


\section{Diana Albanese}

Contadora Pública y Magister en Administración (UNS). Profesora Titular de Auditoría, Directora de la Especialización en Contabilidad y Auditoría (DCA- UNS), Bahía Blanca, Argentina. Docente investigador categoría 2.

\section{dalbanese@uns.edu.ar}

\section{Matías Villegas}

Licenciado en Administración y estudiante avanzado de Contador Público (DCA-UNS). Colaborador en Proyectos de Investigación en el DCA-UNS, Bahía Blanca, Argentina.

matias.villegas@uns.edu.ar 